\title{
AN ANALOGUE IN CERTAIN UNIQUE FACTORIZATION DOMAINS OF THE EUCLID-EULER THEOREM ON PERFECT NUMBERS
}

\section{WAYNE L. MCDANIEL}

Department of Mathematics and Computer Science

University of Missouri-St. Louis

St. Louis, MO, U. S. A. 63121

(Received July 14, 1988)

\begin{abstract}
We show that there exists a natural extention of the sum of divisors function to all unique factorization domains $\mathrm{F}$ having a finite number of units such that if a perfect number in $\mathrm{F}$ is defined to be an integer $\eta$ whose proper divisors sum to $\eta$, then the analogue of Euclid's theorem giving the sufficient condition that an integer be an even perfect number holds in $\mathrm{F}$, and an analogue of the Euclid--Euler theorem giving the necessary and sufficient condition that an even integer be perfect holds in those domains having more than two units, i. e., in $Q(\sqrt{-1})$ and $\mathrm{Q}(\sqrt{-3})$.
\end{abstract}

KEY WORDS AND PHRASES. Sum of divisors, perfect numbers, unique factorization domain.

1980 AMS SUBJECT CLASSIFICATION CODE (1985 Revision). 11R04

\section{INTRODUCTION.}

The usual criterion that a concept has been appropriately defined in an algebraic extension $K$ of the rational number field $Q$ is that the concept's most important characteristics and properties in $\mathrm{Q}$ have their counterpart in $\mathrm{K}$. By this standard, the sum of divisors function has never been satisfactorily extended to an algebraic number field. The function is, of course, easily defined; however, the literature contains no attempts to define the concept in any algebraic number field other than $\mathrm{Q}(\sqrt{-1})$, and this is almost certainly due to the difficulties experienced in proving the analogues in $Q(\sqrt{-1})$ of the best-known theorems involving the sum of divisors function. The sum of divisors function, in this respect, stands apart from the other familiar number-theoretic functions. The Euler phi-function and the number of divisors function, for example, are readily extended to a unique factorization domain having a finite number of units, for they are simply counting functions, and, the Moebius function was defined in $Q(\sqrt{-1})$ at the turn of the century (in 1901 by Gegenbauer [1] (see Dickson [2], vol. 1, p. 447)). The analogue of each of the best-known results involving these functions, including, of course, the generalized Fermat theorem and the Moebius inversion formula have been shown to hold in those fields in which these functions have been defined.

The most widely-known theorem involving the sum of divisors function, apart, possibly, from the theorem establishing the multiplicative nature of the function, is the Euclid-Euler theorem characterizing the even perfect numbers in $\mathrm{Q}$ : 
THEOREM E-E. A rational integer $\mathrm{n}$ is an even perfect number iff there exist rational primes $\mathrm{p}$ and $2^{\mathrm{p}}-1$ such that $\mathrm{n}=2^{\mathrm{p}-1}\left(2^{\mathrm{p}}-1\right)$.

It is the analogue of this theorem which reason would suggest must be provable if an extension of the sum of divisors concept to an algebraic number field is to be accepted as valid and appropriate. Indeed, nearly all researchers who have examined this problem and have obtained publishable results have considered the existence of perfect numbers in the field.

That the analogue of Theorem $\mathrm{E}-\mathrm{E}$ has not been proven in an algebraic extension of $\mathrm{Q}$ (this is not quite true- see our comments below) is related to two problems which arise.

The first of these is that each of the concepts "positive", "sum of divisors", "Mersenne number", "perfect number", and "even" must have a counterpart in the algebraic extension of $\mathrm{Q}$ which is reasonable and "natural" in some sense. A moment's reflection reveals that there may be several reasonable ways to define each of these concepts, so that many combinations of the definitions are possible. This problem was discussed by Spira [3] whose definitions in $Q(\sqrt{-1})$ of "s,um of divisors" and "Mersenne number" we have used in constructing our definitions in this paper. Spira proved an analogue of Euclid's theorem stating the well-known sufficient condition that an even integer be a perfect number; using Spira's definitions, the author of this paper subsequently proved [4] an analogue of Euler's converse, subject to the restriction that the perfect numbers considered are primitive, i. e., not divisible by any other perfect number. (All perfect numbers in $\mathrm{Q}$ are, of course, primitive.) While these results come close to meeting the criteria that the concepts have been appropriately defined in an algebraic extension of $Q$, they appear to fail in one important respect. As W. D. Geyer [5] has properly mentioned in his review of Hausmann and Shapiro's article [6], "Spira has generalized the notion of a perfect number to elements of $\mathrm{Z}[\mathrm{i}]$ in a certain artificial way...". A perfect number had been defined as one whose divisor sum equals the product of the prime of least norm and the number itself. However, there is implicit in Spira's definition a relationship which makes his definition of perfect number much less artificial than might appear. We will discuss this point more fully in section 5 .

The second problem encountered by the researchers who have examined the question of whether an analogue of Theorem E-E can be proved in $Q(\sqrt{-1})$, and which is, in fact, encountered in any unique factorization domain $\mathrm{K}$ having a finite number of units, is that Euler's proof $([7]$, p. 88 , or see $[8])$ that all perfect numbers are of the form $2^{\mathrm{p}-1}\left(2^{\mathrm{p}}-1\right)$ for primes $\mathrm{p}$ and $2^{\mathrm{p}}-1$, and the variation of Euler's proof (apparently due to Dickson [9]) which appears in most introductory number theory texts, do not generalize to $\mathrm{K}$. This is related to the fact that the sum of the divisors of a rational integer exceeds any partial sum of its divisors, whereas in $\mathrm{K}$, the sum $\sigma$ of the divisors of an integer may be "closer" to zero than a partial sum of its divisors. (This is under the assumption that $\sigma$ is a mapping from $\mathrm{K}$ into K.) As an example, using Spira's definition of $\sigma$ in $\mathrm{Q}(\sqrt{-1})$ (see our Definition 2), $\left|\sigma\left((1+2 \mathrm{i})^{2}\right)\right|=\sqrt{37}$, whereas $\left|\sigma\left((1+2 \mathrm{i})^{2}\right)-1\right|=\sqrt{40}$.

In this paper, we overcome each of these difficulties to show that there exists a very nice - and very natural-extension of the sum of divisors function and the other concepts mentioned above to all unique factorization domains having a finite number of units which yields the analogue of Euclid's theorem stating the sufficient condition that an integer be a perfect 


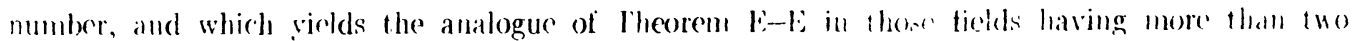
units (i. e. in $Q(\sqrt{-1})$ and $Q(\sqrt{--3}))$. Resolution of the lat.t problem mentioned above involves cxamining the reasonable alternatives to our definitions and showing that they (annot frad to an analogue of Theorem E-E. The second problem is resolued by fist improving a result of Mitrinovic to obtain an inequality which reduces. in the teld of rational integers, to the well-known inequality $\sigma\left(p^{t}\right) / p^{t} \geq(p+1) / p$, for $p$ prime, often used in lesearch on odd perfect numbers; this inequality is then used in obtaining the desired renult.

\section{2. 'TIES DEFINITIONS.}

Let $\mathrm{F}$ be a unique factorization domain (UFD) which has at most a finite number of units. This requirement implices that $F$ is $Q$, or $Q(\sqrt{1})$ for $I)=-1,-2,-3,-7 .-11,-19,-43$, $-67,01-163$. If $F \neq Q$, and $a=a+b \sqrt{D}$ is in $F$, the conjugate $a-b \sqrt{ } \bar{l}$ is denoted by $\alpha^{*}$, and the norm $\|\alpha\|$ of $\alpha$ is the rational integer $\alpha \alpha^{*}$. If $\mathrm{F}=Q$. the conjugatc of a $\in \mathrm{F}$ is at itself, and $\|\mathrm{a}\|=\mathrm{a}^{2}$.

We shall state all the definitions and our main results in this section and discuss these definitions in the next section In our first definition, we select a subsen of the integers in $F$ containing exactly one associate of each integer in $\mathrm{F}$.

DEFINITION 1. Let $\because$ be the mumber of units in $F$. If $F=Q$. $P$ denotes the set of positive integers. If $\mathrm{F} \neq \mathrm{Q}, \mathrm{P}$ drnotes the set of integers $\alpha$ in $\mathrm{F}$ such that $0 \leq \mathrm{arg} \alpha<2 \pi / \mathrm{v}$. The set consisting of the conjugates of the elements of $\mathrm{P}$ is denoted by $\mathrm{P}^{*}$.

Let $\eta \neq 0$ be any integer in $\mathrm{F}$, and, be the unique unit in $\mathrm{F}$ such that for primes $\pi_{1}, \ldots, \pi_{\mathrm{t}}$ in $\mathrm{P}, \eta=\epsilon \Pi \pi_{\mathrm{i}} \mathrm{k}_{\mathrm{i}}$.

DEFINITION 2. 'The sum $\sigma$ of divisors of $\eta$ is defined multiplicatively by

$$
\sigma(\eta)=\Pi \frac{\pi_{\mathrm{i}}^{\left(k_{\mathrm{i}}+1\right)}-1}{\pi_{\mathrm{i}}-1} .
$$

DEFINITION 3. With the exreption of $11 \pi_{\mathrm{i}}{ }^{\mathrm{i}}=\eta / \mathrm{k}$, the lems of the product $\Pi\left(1+\pi_{\mathrm{i}}+\ldots+\pi_{\mathrm{i}} \mathrm{k}_{\mathrm{i}}\right)$ are said to be the proper divisors of $\eta$

DEFINITION 4. The integer $\eta$ is a perfert number if the sum of the proper divisors of $\|$ equals $\eta$.

It is not necessary to define even intcger in $F$ (see Section 7 for additional comments on this point): instead, it suffices to partition the perfect numbers into two classes. Let $\tau$ be a prime in $P$ such that $\tau-1$ is a unit. We note that $F$ contains a unique prime in $P$ of the form $" 1+$ a unit" except when $F=Q(\sqrt{-3})$, in which case both 2 and $\frac{3+\sqrt{-3}}{2}$ are of this form.

DEFINITION 5 . The perfect number $\eta=\left(\| \pi_{\mathrm{j}}{ }^{\mathrm{k}_{\mathrm{i}}}, \pi_{\mathrm{i}} \in \mathrm{P}\right.$. is said to be an E-perfect number in $\mathrm{F}$ if $\tau=\pi_{\mathrm{i}}$ for some $\mathrm{i}$. If $\tau \neq \pi_{\mathrm{i}}$ for any $\mathrm{i}, \|$ is said to be an $\mathrm{O}-$ pes fect number. 
DEFINITION 6. The integer $\sigma\left(\tau^{\mathrm{k}-1}\right)=\left(\tau^{\mathrm{k}}-1\right) /(\tau-1)=\mathrm{M}_{\mathrm{k}}$ is called an F-Mersenne number; if $\mathrm{M}_{\mathrm{k}}$ is prime in $\mathrm{F}, \mathrm{M}_{\mathrm{k}}$ is called an $\mathrm{F}$-Mersenne prime.

DEFINITION 7. The perfect number $\eta$ is said to be primitive if there exists no perfect number $\alpha \neq \eta$ such that $\alpha \mid \eta$. A perfect number which is not primitive is said to be imprimitive.

The main results of this paper are the following analogues of the Euclid and Euler theorems characterizing the even perfect numbers.

THEOREM 1. (Analogue of Euclid's theorem). Let $M_{p}$ be an $F-$ Mersenne prime. If $\mathrm{M}_{\mathrm{p}} \in \mathrm{P}^{*}, \eta=\tau^{\mathrm{p}-1} \mathrm{M}_{\mathrm{p}}$ is a perfect number.

THEOREM 2. (Analogue of Thm. E-E). Let $\mathrm{F}$ be $\mathrm{Q}, \mathrm{Q}(\sqrt{-1})$, or $\mathrm{Q}(\sqrt{-3})$, and $\mu$ be an integer in $\mathrm{F}$. The integer $\eta=\tau^{\mathrm{k}-1} \mu$ is a primitive E-perfect number if and only if $\mathrm{k}$ is a rational prime, and $\mu$ is an $\mathrm{F}-$ Mersenne prime in $\mathrm{P}^{*}$. If $\mathrm{F}$ is $\mathrm{Q}$ or $\mathrm{Q}(\sqrt{-3})$, there exist no other $\mathrm{E}-$ perfect numbers, i. e., all E-perfect numbers are primitive.

\section{DISCUSSION OF THE CONCEPTS DEFINED IN SECTION 2.}

The reader will note that if $\mathrm{F}$ is $\mathrm{Q}$, Definitions 1 through 6 are the familiar definitions of the corresponding concepts in $\mathrm{Q}$ provided $\mathrm{E}$-perfect and $\mathrm{O}$-perfect are read as even perfect and odd perfect, in Definition 5 (except that in addition to defining $\sigma$ in the usual way if $\eta$ is a positive rational integer, we have, also, defined $\sigma$ for $\eta$ negative in Definition 2). Theorem 1 is Euclid's theorem in Q, and Theorem 2 is Theorem E-E. While these definitions are, for the most part, quite natural, alternate definitions are possible, and some have been employed with partial success in previous investigations. The rationale for our choices goes beyond the desire to extend the concepts in a natural way, however, and we shall see that alternate reasonable choices for the definitions do not lead to fully satisfactory analogues of Theorem E-E.

The Set $P$. In $\mathrm{Q}(\sqrt{-1})$ (whose units are $\pm 1, \pm \mathrm{i}$ ), $\mathrm{P}$ has been defined to be the first quadrant of the complex plane including the positive half of the real axis and excluding the imaginary axis, and in $Q(\sqrt{-3})$ (whose units are $\pm 1, \frac{1 \pm \sqrt{-3}}{2}$ and $\frac{-1 \pm \sqrt{-3}}{2}$ ), $\mathrm{P}$ is the first sextant of the complex plane including the positive half of the real axis and excluding the axis $y=\sqrt{3} x$; all remaining fields have only the units 1 and -1 , and in these fields $P$ is the upper half-plane including the positive but not the negative real axis.

It will be seen in Section 6 that an analogue of Euler's converse of Euclid's theorem requires that the primes $\pi$ in $\mathrm{P}$ satisfy the condition that $\left\|\sigma\left(\pi^{\mathrm{k}}\right) / \pi^{\mathrm{k}}\right\|>1$. It is well-known that this inequality holds in $Q$, and Mitrinovic ([10], p. 140) proved that it holds for $\alpha$ any complex number such that $\operatorname{Re} \alpha \geq 1$; it, in fact, fails to hold for infinitely many powers of each complex number $\alpha$ such that $\operatorname{Re} \alpha=\mathrm{c}<1$. Our definition of $\mathrm{P}$ assures that in $\mathrm{Q}(\sqrt{-1})$ and $\mathrm{Q}(\sqrt{-3})$ this inequality holds for $\pi \in \mathrm{P}$; If $\mathrm{F} \neq \mathrm{Q}, \mathrm{Q}(\sqrt{-1})$, or $\mathrm{Q}(\sqrt{-3})$, no choice for $\mathrm{P}$ such that $\pi \in \mathrm{P} \Rightarrow\left\|\sigma\left(\pi^{\mathrm{k}}\right) / \pi^{\mathrm{k}}\right\|>1$ is possible, since, in each of these fields, there exist integers both of whose associates have their real part $<1$ (suggesting the reason for the failure of the Euler converse for these fields). Definition 1 was motivated, also, by the fact that in $Q(\sqrt{-3}), P$ 
contains two primes $\tau$ such that $\tau-1$ is a unit-the validity of the analoguc of Theorem E-E for each of the primes $\tau$ demonstrates the essential nature of the concept "even", as we have extended it.

An alternate choice for $P$ which has been made in at least one investigation of perfect numbers in $Q(\sqrt{-1})$ (Randall, [11]) involved choosing $P$ so that the positive real axis is an axis of symmetry for $P$. With $P$ so defined, however, it is not possible to prove the analogue of Theorem $\mathrm{E}-\mathrm{E}$ in either $\mathrm{Q} \sqrt{-1}$ or $\mathrm{Q}(\sqrt{-3})$ : In order to show that $\eta$ is a perfect number of the form $\eta=\epsilon \alpha^{\mathrm{k}-1} \beta$, for $\epsilon$ a unit and and $\alpha$ and $\beta$ prime in $\mathrm{P}$, one can show that $\alpha$ must be $1+\epsilon^{-1}$ and $\beta=\alpha^{k}-1$. However, in $\mathrm{Q} \sqrt{-1}, 1+\epsilon^{-1}$ is a prime in $\mathrm{P}$ only if $\epsilon^{-1}=\mathrm{i}$, implying that $\beta=2^{\mathrm{k} / 2}\left(\cos \frac{\pi}{4} \mathrm{k}+\mathrm{i} \sin \frac{\pi}{4} \mathrm{k}\right)-1$; but, then, $\beta \in \mathrm{P}$ only if $\mathrm{k} \equiv 0(\bmod 8)$, and $\beta$ prime implies that, in addition, $k$ is a rational prime, which is clearly not possible. Similarly, in $Q \sqrt{-3}$, $1+\epsilon^{-1}$ is a prime in $\mathrm{P}$ only if $\epsilon^{-1}=1$ or $(1+\sqrt{-3}) / 2$, and in the latter case, we have $\beta=\alpha^{\mathrm{k}}-1=3^{\mathrm{k} / 2}\left(\cos \frac{\pi}{6} \mathrm{k}+\mathrm{i} \sin \frac{\pi}{6} \mathrm{k}\right)-1$ which implies, as above, that $\mathrm{k} \equiv 0(\bmod 12)$, so that, again, $\beta$ is not prime; it follows that if $\tau=(3+\sqrt{-3}) / 2, \quad \mathrm{Q}(\sqrt{-3})$ has no E-perfect numbers of the familiar form specified in Theorem 2. Randall showed, for this choice of $P$, that if a perfect number in $\mathrm{Q}(\sqrt{-1})$ is defined by $\sigma(\eta)=2 \eta$, then all perfect numbers in $\mathrm{Q}(\sqrt{-1})$ have at least three prime factors and 5 is the only perfect number $<10^{6}$.

The Sum of Divisors Function. Previous investigations of problems involving the sum of divisors function in $Q(\sqrt{-1})$ have used the concept as defined in Definition 2 [3], [4], [11] (although not all have required that the prime factors of $\eta$ be in the set $\mathrm{P}$ as we have defined it), or, have defined $\sigma$ to be the sum of the norms of the divisors of $\eta[12]$. In related work, Hausmann and Shapiro [6] investigated perfect ideals over the Gaussian integers, defining the sum of divisors of an ideal to be the sum of the norms of the ideal's divisors; in this paper, the authors found only two perfect ideals and showed that there are no others having fewer than five distinct prime factors.

Perfect Number. Definition 4 is, of course, the classical definition of a perfect number. In view of Definition 3, $\eta=\epsilon \Pi \pi_{\mathrm{i}}$ is perfect if $\sigma(\eta)-\eta / \epsilon=\eta$, that is, if $\sigma(\eta)=\left(1+\epsilon^{-1}\right) \eta$. We note that if $\eta \in Q, \epsilon^{-1}$ is 1 , and, clearly, this relation corresponds to the familiar condition that $\sigma(\eta)=2 \eta$.

Even Perfect Number. Our decision to classify the perfect numbers as E-perfect numbers or $\mathrm{O}$-perfect numbers as opposed to defining an integer $\eta$ in $\mathrm{F}$ as even iff $\tau \mid \eta$ is related to the fact that the laws of parity do not necessarily hold in $\mathrm{F}$ under this definition. They do hold if $F \neq Q(\sqrt{-3})$; however, it is not difficult to construct examples to show that if $F=Q(\sqrt{-3})$ and $\tau=(3+\sqrt{-3}) / 2$, the sum of two odd integers may be odd. There is, on the other hand, no requirement that the partitioning of perfect numbers into two classes satisfies the laws of parity-the sum of two even perfect numbers in $Q$, for example, is not an even perfect number.

The concept of "evenness" in F may be defined by any one of the following:

(i) $\mathrm{n}$ is even if $2 \mid \mathrm{n}$;

(ii) $\mathrm{n}$ is even if $\mathrm{n}$ is divisible by the prime factor of 2 of least norm;

(iii) $\mathrm{n}$ is even if $\mathrm{n}$ is divisible by the prime in $\mathrm{F}$ of least norm;

(iv) $n$ is even if $n$ is divisible by a prime of the form $1+u$ where $u$ is a unit. 
While these definitions ate equivalent in $Q$, it is todily seen that they ane not equivalent in, for example, $Q(\sqrt{-1})$ and $Q(\sqrt{-3})$. We have not chosen to extend the conceph of "evenness" by using any of the first three definitions because, in each (ase, the analogue of Iheorem $\mathrm{E}-\mathrm{E}$ : fails in one or more fields $F$ (it fails in $Q(\sqrt{-1})$ for (i), and in $Q(\sqrt{-2})$ for (ii) and (iii), for instance). The concept even integer in an algebraic number ficld will be discussed funther in Section 7.

F-Mersenne Number. The definition is identical to the definition of Messenne number in Q. As in $\mathrm{Q}, \mathrm{M}_{\mathrm{k}}$ is a prime in $\mathrm{F}$ only if $\mathrm{k}$ is a rational prime

\section{TIIE PROOF OF THEOREM 1 AND A LOWER BOUNI) FOR $\left\|\sigma\left(\pi^{t}\right) / \pi^{t}\right\|$.}

Theorem 1 is readily established.

PROOF OF THEOREM 1. By definition, $M_{p}=\sigma\left(\tau^{p-1}\right)=\left(\tau^{p} \cdots 1\right) /(\tau-1)$. Il'nce. $\eta=\tau^{\mathrm{p}-1} \mathrm{M}_{\mathrm{p}}$ implies that

$$
\sigma(\eta)=\sigma\left(\tau^{\mathrm{p}-1}\right) \sigma\left(\mathrm{M}_{\mathrm{p}}\right)=\mathrm{M}_{\mathrm{p}} \sigma\left(\mathrm{M}_{\mathrm{p}}\right)
$$

Now, if $\tau-1=1, \mathrm{M}_{\mathrm{p}}=2^{\mathrm{p}}-1$ is in $\mathrm{P} ; \quad$ if $\tau-1 \neq 1, \tau-1=\mathrm{i} \quad$ (if $\eta \in \mathrm{Q}(\sqrt{-1})$ ), o1 $\tau-1=(1+\sqrt{-3}) / 2$ (if $\eta \in Q(\sqrt{-3})$ ). In either case, since, by hypothesis, $\mathrm{M}_{\mathrm{p}} \in \mathrm{P}^{*}$, the associate of $\mathrm{M}_{\mathrm{p}}$ in $\mathrm{P}$ is $\mathrm{M}_{\mathrm{p}}(\tau-1)=\tau^{\mathrm{P}}-1$. Hence,

$$
\sigma(\eta)=\mathrm{M}_{\mathrm{p}} \sigma\left(\mathrm{M}_{\mathrm{p}}\right)=\mathrm{M}_{\mathrm{p}} \sigma\left(\tau^{\mathrm{p}}-1\right)=\mathrm{M}_{\mathrm{p}} \tau^{\mathrm{p}}=\tau \eta . \quad \text { Q.E.D. }
$$

In order to prove Theorem 2, we first obtain an inequality which reduces to the woll--known lower bound often used in research on odd perfect numbers:

$$
\sigma\left(\mathrm{p}^{\mathrm{t}}\right) / \mathrm{p}^{\mathrm{t}} \geq(\mathrm{p}+1) / \mathrm{p}
$$

where equality holds iff $\mathrm{t}=1$.

Mitrinovic ([10], p. 140) published a proof that if $z$ is an arbitrary complex number with Re $z \geq 1$, then $\left\|\left(z^{t+1}-1\right) /(z-1)\right\|$ is bounded below by $\left\|z^{t}\right\|$. We now improve upon this lower bound for $\operatorname{Re} z \geq 5 / 4$.

THEOREM 3. Let $\mathrm{z}=\mathrm{x}+\mathrm{iy}=\mathrm{re}^{\mathrm{i} \theta}$, with $\mathrm{x} \geq 5 / 4$. If $\mathrm{t}$ is a positive integer,

$$
\left\|1+z+\ldots+z^{t}\right\|>\left\|z^{t-1}\right\| \cdot(\|z\|+2 x-1),
$$

and. moreover, if $|y| \leq x-1$,

$$
\left\|1+z+\ldots+z^{t}\right\| \geq\left\|z^{t-1}\right\| \cdot(\|z\|+2 x+1),
$$

with equality holding iff $\mathrm{t}=1$.

PROOF. If $\mathrm{t}=1,\|1+\mathrm{z}\|=(1+\mathrm{x})^{2}+\mathrm{y}^{2}=\|\mathrm{z}\|+2 \mathrm{x}+1$. 
If $\mathrm{t}=2$,

$$
\begin{aligned}
\left\|1+z+z^{2}\right\| & =\|z\| \cdot\left\|z^{-1}+1+z\right\| \\
& =\|z\| \cdot\left[\|z\|+2 x+1+\left(2 x^{2}+2 x+1-2 y^{2}\right) /\|z\|\right] \\
& >\left\{\begin{array}{l}
\|z\| \cdot(\|z\|+2 x-1) \text { for all } y, \\
\|z\| \cdot(\|z\|+2 x+1) \text { for }|y| \leq x .
\end{array}\right.
\end{aligned}
$$

If $\mathrm{t} \geq 3$,

$$
\begin{aligned}
\left\|1+z+\ldots+z^{t}\right\| & =\left\|z^{t}\right\| \cdot\left[r^{2}+r^{-2 t}-2 r^{1-t} \cos (t+1) g /\left(r^{2}-2 x+1\right)\right. \\
& >\left\|z^{t}\right\| \cdot\left(r^{2}-2 r^{-2}\right) /\left(r^{2}-2 x+1\right) .
\end{aligned}
$$

Supposing that $\left(r^{2}-2 r^{-2}\right) /\left(r^{2}-2 x+1\right)$ is less than $\left(r^{2}+2 x-1\right) / r^{2}$, and then less than $\left(r^{2}+2 x+1\right) / r^{2}$ when $|y|<x-1$, leads to the desired contradictions.

COROLLARY. If $\pi$ is a prime in $\mathrm{Q}(\sqrt{-3})$ and $\mathrm{x}+\mathrm{y} \sqrt{-3}$ is the associate in $\mathrm{P}$ of $\pi$,

$$
\left\|\sigma\left(\pi^{\mathrm{t}}\right) / \pi^{\mathrm{t}}\right\|>(\|\pi\|+2 \mathrm{x}-1) /\|\pi\|
$$

if $\mathrm{y} \leq \mathrm{x}-1$,

$$
\left\|\sigma\left(\pi^{\mathrm{t}}\right) / \pi^{\mathrm{t}}\right\| \geq(\|\pi\|+2 \mathrm{x}+1) /\|\pi\|,
$$

with equality holding iff $t=1$.

PROOF. Since $\|\pi\|=\|\mathrm{x}+\mathrm{y} \sqrt{-3}\|$ and $\left\|\sigma\left(\pi^{\mathrm{t}}\right)\right\|=\left\|1+\ldots+(\mathrm{x}+\mathrm{y} \sqrt{-3})^{\mathrm{t}}\right\|$, the corollary is merely a restatement of the theorem for $z=\pi$ a prime in $Q(\sqrt{-3})$, provided we show that $x \geq 5 / 4$.

Because $x+y \sqrt{-3}$ is an integer in $Q(\sqrt{-3}), x+y \sqrt{-3}=(a+b \sqrt{-3}) / 2$, where $a$ and $b$ are rational integers of the same parity. Since this integer is in $P, b<a$, and it is therefore immediate that $x=a / 2$ is not $1 / 2$ nor 1 . So, $x \geq 3 / 2>5 / 4$.

If $\mathrm{z}=\mathrm{p}, \mathrm{a}$ rational prime, the second inequality of Theorem 3 (or of the Corollary) becomes the bound $\sigma\left(\mathrm{p}^{\mathrm{t}}\right) / \mathrm{p}^{\mathrm{t}} \geq(\mathrm{p}+1) / \mathrm{p}$.

\section{E-PERFECT NUMBERS IN $Q \sqrt{-1}$.}

We have stated, in the Introduction, that while Spira defined an integer $\eta$ in $\mathrm{Q}(\sqrt{-1})$ to be a perfect number if $\sigma(\eta)=\tau_{0} \eta$, where $\tau_{0}$ is the prime in $\mathrm{P}$ of least norm (that is, $\left.\tau_{0}=1+\mathrm{i}\right)$, this definition, in fact, implies a more fundamental relationship. We shall show that in this section, and prove Theorem 2 for $F=Q(\sqrt{-1})$.

Our Definitions 1, 2 and 6 (for $F=Q(\sqrt{-1})$ ) are those used by Spira in defining P, "sum of divisors" and "Mersenne number" in $Q(\sqrt{-1})$, and Spira defined the integer $\eta$ to be even if $(1+i) \mid \eta$. Incorporating Spira's proof of the sufficiency in a theorem characterizing the even perfect numbers in $Q(\sqrt{-1})$, under the above definitions, the author proved [4]

THEOREM A. $\eta$ is an even primitive perfect number iff there exists a rational prime $\mathrm{p} \equiv 1(\bmod 8)$ and a Mersenne prime $\mathrm{M}_{\mathrm{p}}$ such that $\eta=(1+\mathrm{i})^{\mathrm{p}-1} \mathrm{M}_{\mathrm{p}}$. 
We shall show that Theorem A, under Spira's definitions of even integer and perfect number, is equivalent to our analogue of Theorem $\mathrm{E}-\mathrm{E}$, i. e., to Theorem 2 with $\mathrm{F}=\mathrm{Q}(\sqrt{-1})$.

Our definition of $\mathrm{P}$ and $\tau$ in $\mathrm{Q}(\sqrt{-1})$ imply that $\tau=1+\mathrm{i}=\tau_{0}$, so, in Theorem $\mathrm{A}, \eta$ is equal to $\tau^{\mathrm{p}-1} \mathrm{M}_{\mathrm{p}}$. Since

$$
\mathrm{M}_{\mathrm{p}}=\left(\tau^{\mathrm{p}}-1\right) /(\tau-1)=\left[2^{\mathrm{p} / 2}\left(\cos \frac{\pi}{4} \mathrm{p}+\mathrm{i} \sin \frac{\pi}{4} \mathrm{p}\right)-1\right] / \mathrm{i}
$$

it is readily seen that the condition that $p \equiv 1(\bmod 8)$ is equivalent to $M_{p} \in P^{*}$. Finally, under Spira's definitions, $\eta=\epsilon \tau^{\mathrm{p}-1} \mu$, for $\epsilon$ a unit and $\mu$ the product of prime factors in $\mathrm{P}$, is an even perfect number iff $\sigma(\eta)=(1+\mathrm{i}) \eta$, and, by the observation in Section 3 concerning our Definition $4, \eta$ is E-perfect iff $\sigma(\eta)=\left(1+\epsilon^{-1}\right) \eta$. It is now clear that Theorem A and Theorem 2 with $\mathrm{F}=\mathrm{Q}(\sqrt{-1})$ are equivalent if $\epsilon=-\mathrm{i}$. But this is indeed the case, for, upon observing that the associate in $P$ of $M_{p}$ is $M_{p}^{\prime}=i M_{p}$, we see that the perfect number $\eta=(1+i)^{p-1} M_{p}$, in Theorem $\mathrm{A}$, can be written $\eta=-\mathrm{i} \tau^{\mathrm{p}-1} \mathrm{M}_{\mathrm{p}}^{\prime} \quad\left(\mathrm{M}_{\mathrm{p}}^{\prime} \in \mathrm{P}\right)$; therefore $\eta$ is of the form $\eta=\epsilon \tau^{\mathrm{p}-1} \mu$, with $\epsilon=-\mathrm{i}$.

\section{E-PERFECT NUMBERS IN $Q(\sqrt{-3})$.}

In this section, we shall complete the proof of Theorem 2 .

The Form of E-Perfect Numbers in $Q(\sqrt{-3})$. In the proof of the following lemma, we let $\mathrm{N}_{\mathrm{k}}=\left\|\mathrm{M}_{\mathrm{k}}\right\|$.

LEMMA 1. If, for $(\tau, \mu)=1$ and $\mathrm{k}>1, \eta=\tau^{\mathrm{k}-1} \mu$ is a perfect number in $\mathrm{Q}(\sqrt{-3})$, then the $\mathrm{Q}(\sqrt{-3})-$ Mersenne number $\mathrm{M}_{\mathrm{k}}=\sigma\left(\tau^{\mathrm{k}-1}\right)$ is prime.

PROOF. Assume $\eta=\tau^{\mathrm{k}-1} \mu$ is a perfect number in $\mathrm{Q}(\sqrt{-3})$. Let $\pi=\mathrm{x}+\mathrm{y} \sqrt{-3}$ be any prime factor in $\mathrm{P}$ of $\mathrm{M}_{\mathrm{k}}$. Since $\left(\tau, \mathrm{M}_{\mathrm{k}}\right)=1$,

$$
\tau \eta=\sigma(\eta)=\sigma\left(\tau^{\mathrm{k}-1}\right) \sigma(\mu)=\mathrm{M}_{\mathrm{k}} \sigma(\mu)
$$

implies that $\pi \mid \eta$, and hence, $\mu$; we let a be the largest rational integer such that $\pi^{\mathrm{a}} \mid \mu$. Since, by the Corollary to Theorem $1,\left\|\sigma\left(\alpha^{\mathrm{t}}\right) / \alpha^{\mathrm{t}}\right\|>1$ for any prime power $\alpha^{\mathrm{t}}$, we have, upon using the multiplicative property of $\sigma$,

$$
1=\|\sigma(\eta) / \tau \eta\| \geq\left\|\sigma\left(\tau^{\mathrm{k}-1}\right) \sigma\left(\pi^{\mathrm{a}}\right) / \tau^{\mathrm{k}} \pi^{\mathrm{a}}\right\|>\mathrm{N}_{\mathrm{k}}(\|\pi\|+2 \mathrm{x}-1) /\left\|\tau^{\mathrm{k}}\right\| \cdot\|\pi\| .
$$

In the proof of the Corollary to Theorem 3, we observed, for $\pi=x+y \sqrt{-3}$, that $x \geq 3 / 2$. Since the only prime in $\mathrm{P}$ having its real part equal to $3 / 2$ is $\tau, \mathrm{x} \geq 2$; hence, applying the Corollary to Theorem 3 to the above inequality,

$$
\|\pi\|>\mathrm{N}_{\mathrm{k}}(2 \mathrm{x}-1) /\left(\|\tau\|^{\mathrm{k}}-\mathrm{N}_{\mathrm{k}}\right) \geq 3 \mathrm{~N}_{\mathrm{k}} /\left(\|\tau\|^{\mathrm{k}}-\mathrm{N}_{\mathrm{k}}\right) .
$$


Suppose $3 N_{k} /\left(\|\tau\|^{k}-N_{k}\right)<N_{k}^{1 / 2}$. Now, if $\tau=2 . \quad N_{k}=\left(2^{k}-1\right)^{2}$, and if $\tau=(3+\sqrt{-3}) / 2$. $M_{k}=\left[3^{k / 2}\left(\cos \frac{\pi}{6} k+i \sin \frac{\pi}{6} k\right)-1\right] /(\tau-1)$, implying that

$$
N_{k}=\left(\tau^{k}-1\right)\left[\left(\tau^{k}\right)^{*}-1\right]=3^{k}-2 \cdot 3^{k / 2} \cdot \cos \frac{\pi}{6} k+1
$$

Substituting and simplifying, we find that, for each value of $T$, the resulting inequality does not. hold for $k>1$. Since $M_{k}$ is a factor of $\eta$, it follows that $\|\pi\|>N_{k}^{1 / 2}=\left\|M_{k}\right\|^{1 / 2}$; hence, for some unit $\epsilon, \pi=\epsilon \mathrm{M}_{\mathrm{k}}$, implying that $\mathrm{M}_{\mathrm{k}}$ is prime.

THEOREM 4. If $\eta$ is an E-perfect number in $Q(\sqrt{-3})$, then there exists a $\mathrm{Q}(\sqrt{-3})$-Mersenne prime, $\mathrm{M}_{\mathrm{p}}$ in $\mathrm{P}^{*}$ such that for some positive rational integer $1 \geq 1$ and integer $\delta$ such that $(\tau, \delta)=1, \quad \eta=\tau^{\mathrm{p}-1} \mathrm{M}_{\mathrm{p}}^{\mathrm{t}} \delta$.

PROOF. Since $\mathrm{M}_{\mathrm{k}}$ is a factor of $\eta$, Lemma 1 inplies that an E-perfect number in $\mathrm{Q}(\sqrt{-3})$ has the form specified in the theorem, except that it has not been shown that $M_{p} \in \mathrm{P}^{*}$ when $\tau=(3+\sqrt{-3}) / 2$ (when $\tau=2, \mathrm{M}_{\mathrm{k}}=2^{\mathrm{k}}-1 \in \mathrm{P}=\mathrm{P}^{*}$ ). Assume that $\tau=(3+\sqrt{-3}) / 2$ and that $\eta=\tau^{\mathrm{p}-1} \mu$ is a perfect number in $Q(\sqrt{-\overline{3}})$. Then,

implies

$$
\left\|\tau^{\mathrm{p}} \mu\right\|=\|\tau \eta\|=\|\sigma(\eta)\|=\left\|\mathrm{M}_{\mathrm{p}}\right\| \cdot\|\sigma(\mu)\|
$$

$$
\left\|\mathrm{M}_{\mathrm{p}}\right\|=\left\|\tau^{\mathrm{p}}\right\| \cdot(\|\mu\| /\|\sigma(\mu)\|)<\left\|\tau^{\mathrm{p}}\right\|=3^{\mathrm{p}}
$$

Using (6.2), we have

$$
3^{p}-2 \cdot 3^{p / 2} \cdot \cos \frac{\pi}{6} p+1<3^{p}
$$

It is clear that $(6.4)$ holds only if $\cos \frac{\pi}{6} p$ is positive. That is, only if $p \equiv 0, \pm 1$, or $\pm 2(\bmod 12)$. Since $\mathrm{p}$ is a rational prime, $\mathrm{p}$ is not congruent to 0 or \pm 2 , modulo 12 , unless $p=2$. If $p=2$, however, $M_{p}=\tau+1=\frac{5}{2}+\frac{\sqrt{-3}}{2}$, and

$$
\begin{aligned}
1 & =\|\sigma(\eta) / \tau \eta\| \geq\left\|\sigma(\tau) \sigma\left(\mathrm{M}_{2}{ }^{\mathrm{t}}\right) / \tau^{2} \cdot \mathrm{M}_{2}{ }^{\mathrm{t}}\right\| \\
& \geq\left\|\sigma(\tau) \sigma\left(\mathrm{M}_{2}\right)\right\| / 9 \cdot\left\|\mathrm{M}_{2}\right\|=\left\|\sigma\left(\mathrm{M}_{2}\right)\right\| / 9=13 / 9 .
\end{aligned}
$$

Thus, $p \equiv \pm 1(\bmod 12) . \quad$ Suppose $p \equiv-1(\bmod 12) . \quad$ Then

$$
M_{p}=\left(3^{(p+1) / 2}-2-3^{p / 2} i\right) / 2(\tau-1),
$$

and the associate in $\mathrm{P}$ of $\mathrm{M}_{\mathrm{p}}$ is 


$$
\alpha=\left[3^{(\mathrm{p}+1) / 2}-1+\left(3^{\mathrm{p} / 2}-3^{1 / 2}\right) \mathrm{i}\right] / 2 .
$$

It fo'lows, using the Corollary to Theorem 3, that

$$
\begin{aligned}
\|\sigma(\eta) / \tau \eta\| & =\left\|\sigma\left(\tau^{\mathrm{p}-1}\right) \sigma\left(\mathrm{M}_{\mathrm{p}}^{\mathrm{t}}\right) \sigma(\delta) / \tau^{\mathrm{p}} \mathrm{M}_{\mathrm{p}}^{\mathrm{t}} \delta\right\| \\
& \geq\left\|\mathrm{M}_{\mathrm{p}}\right\| \cdot\left(\left\|\mathrm{M}_{\mathrm{p}}\right\|+2 \operatorname{Re} \alpha+1\right) / 3^{\mathrm{p}}\left\|\mathrm{M}_{\mathrm{p}}\right\| \\
& =\left(3^{\mathrm{p}}+1\right) / 3^{\mathrm{p}}>1 .
\end{aligned}
$$

Hence, $\eta$ E-perfect implies that $\mathrm{p} \equiv 1(\bmod 12) ; \quad$ since $\mathrm{p} \equiv 1(\bmod 12)$ implies that $\mathrm{M}_{\mathrm{p}}=\left(3^{(\mathrm{p}+1) / 2}-2+3^{\mathrm{p} / 2} \mathrm{i}\right) / 2(\tau-1), \mathrm{M}_{\mathrm{p}}$ is in $\mathrm{P}^{*}$.

The Non-Existence of Imprimitive E-Perfect Numbers in $Q(\sqrt{-3})$. Imprimitive perfect numbers do not exist in $Q$, but imprimitive perfect numbers may exist in $Q(\sqrt{-1})$ (see [4]). The possibility of the existence of an imprimitive perfect number in an algebraic number field is related to the fact that if $\pi$ is a prime in the field, $\mathrm{f}(\mathrm{t})=\sigma\left(\pi^{\mathrm{t}}\right) / \pi^{\mathrm{t}}$ may not be an increasing function of $t$ (as it is in Q). For example, in $Q(\sqrt{-3})$, if $\pi=1+2 \sqrt{-3}$, $\left\|\sigma\left(\pi^{2}\right) / \pi^{2}\right\|=189 / 169<16 / 13=\|\sigma(\pi) / \pi\|$. It is thus conceivable that if, for example, $\eta=\tau^{\mathrm{p}-1} \mathrm{M}_{\mathrm{p}}$ is a perfect number, then $\left\|\sigma\left(\tau^{\mathrm{p}-1} \mathrm{M}_{\mathrm{p}}{ }^{\mathrm{t}}\right) / \tau^{\mathrm{p}} \mathrm{M}_{\mathrm{p}}{ }^{\mathrm{t}}\right\|<1$ for some $\mathrm{t}$, and there exists an integer $\delta$ such that $\left\|\sigma\left(\tau^{\mathrm{p}-1} \mathrm{M}_{\mathrm{p}}{ }^{\mathrm{t}} \delta\right) / \tau^{\mathrm{p}} \mathrm{M}_{\mathrm{p}}{ }^{\mathrm{t}} \delta\right\|=1$. Indeed, this is precisely the situation in $\mathrm{Q}(\sqrt{-1})$. We shall show, however, that this does not happen in $\mathrm{Q}(\sqrt{-3})$, i. e., that imprimitive E-perfect numbers do not exist in $\mathrm{Q}(\sqrt{-3})$.

LEMMA 2. Let $M_{p}$ be a $Q(\sqrt{-3})$-Mersenne prime in $P^{*}$. The inequality $\left\|\sigma\left(\mathrm{M}_{\mathrm{p}}\right) / \mathrm{M}_{\mathrm{p}}\right\|<\left\|\sigma\left(\mathrm{M}_{\mathrm{p}}{ }^{\mathrm{t}}\right) / \mathrm{M}_{\mathrm{p}} \mathrm{t}^{\mathrm{t}}\right\|$ holds for all rational integers $\mathrm{t} \geq 2$.

PROOF. If $\tau=2$, the lemma is clearly true. Assume $\tau=(3+\sqrt{-3}) / 2$. Suppose the lemma is false, and let $M_{p}^{\prime}=r e^{i \theta}$ denote the associate in $P$ of $M_{p}$. Now,

$$
\left\|\sigma\left(\mathrm{M}_{\mathrm{p}}\right) / \mathrm{M}_{\mathrm{p}}\right\| \geq\left\|\sigma\left(\mathrm{M}_{\mathrm{p}}{ }^{\mathrm{t}}\right) / \mathrm{M}_{\mathrm{p}}{ }^{\mathrm{t}}\right\| \Rightarrow\left\|\mathrm{M}_{\mathrm{p}}{ }^{2}-1\right\| \geq\left\|\mathrm{M}_{\mathrm{p}}^{\mathrm{t}+1}-1\right\| /\left\|\mathrm{M}_{\mathrm{p}}^{\mathrm{t}-1}\right\|
$$

which implies that

$$
r^{4}-2 r^{2} \cdot \cos 2 \theta+1 \geq\left(r^{2 t+2}-2 r^{t+1} \cdot \cos (t+1) \theta+1\right) / r^{2 t-2} .
$$

That is,

$$
\mathrm{r}^{\mathrm{t}-3}-2 \mathrm{r}^{\mathrm{t}-1} \cdot \cos 2 \theta \geq \mathrm{r}^{-\mathrm{t}-1}-2 \cdot \cos (\mathrm{t}+1) 0
$$

Since, for $M_{p} \in P^{*}$,

$$
\begin{gathered}
\left(\mathrm{M}_{\mathrm{p}}^{\prime}\right)^{2}=\left[\left(3^{(\mathrm{p}+1) / 2}-2+3^{\mathrm{p} / 2} \mathrm{i}\right) / 2\right]^{2}=\mathrm{r}^{2}(\cos 2 \theta+\mathrm{i} \sin 20), \\
\cos 2 \theta=\left(3^{\mathrm{p}} / 2-3^{(\mathrm{p}+1) / 2}+1\right) / \mathrm{r}^{2}
\end{gathered}
$$


Substututing in (6.5),

$$
\mathrm{r}^{\mathrm{t}-3}\left(3^{\mathrm{p}}-2 \cdot 3^{(\mathrm{p}+1) / 2}+1\right) \leq 2 \cdot \cos (\mathrm{t}+1) \theta-\mathrm{r}^{-\mathrm{t}-1} .
$$

The left member of this inequality is $\geq 10 \mathrm{r}^{\mathrm{t}-3}$ for $\mathrm{p} \geq 3$; since the right member is negative for $\mathrm{t}=2$ and is less than 2 for $\mathrm{t} \geq 3$, we have a contradiction.

THEOREM 5. There exist no imprimitive E-perfect numbers in $Q(\sqrt{-3})$.

PROOF. Let $\eta$ be an E-perfect number. By Theorem $4, \eta=\tau^{\mathrm{p}-1} \mathrm{M}_{\mathrm{p}}^{\mathrm{t}} \delta$, with $\mathrm{M}_{\mathrm{p}} \in \mathrm{P}^{*}$. Now, using Lemma 2,

$$
\begin{aligned}
1 & =\|\sigma(\eta) / \tau \eta\|=\left\|\sigma\left(\tau^{\mathrm{p}-1}\right) / \tau^{\mathrm{p}}\right\| \cdot\left\|\sigma\left(\mathrm{M}_{\mathrm{p}}{ }^{\mathrm{t}}\right) / \mathrm{M}_{\mathrm{p}}{ }^{\mathrm{t}}\right\| \cdot\|\sigma(\delta) / \delta\| \\
& \geq\left\|\sigma\left(\tau^{\mathrm{p}-1}\right) / \tau^{\mathrm{p}}\right\| \cdot\left\|\sigma\left(\mathrm{M}_{\mathrm{p}}\right) / \mathrm{M}_{\mathrm{p}}\right\| .
\end{aligned}
$$

However, by Theorem 1, the integer $\tau^{\mathrm{p}-1} \mathrm{M}_{\mathrm{p}}$ is a perfect number, so $\left\|\sigma\left(\tau^{\mathrm{p}-1}\right) / \tau^{\mathrm{p}}\right\| \cdot\left\|\sigma\left(\mathrm{M}_{\mathrm{p}}\right) / \mathrm{M}_{\mathrm{p}}\right\|=1$. Strict equality, then, must hold, so $\mathrm{t}=1$ and $\delta$ is a unit, $\epsilon$. Now $\eta=\epsilon \tau^{\mathrm{k}-1} \mathrm{M}_{\mathrm{p}}$, implies that

$$
\sigma(\eta)=\sigma\left(\tau^{\mathrm{p}-1}\right) \sigma\left(\mathrm{M}_{\mathrm{p}}\right)=\mathrm{M}_{\mathrm{p}} \sigma\left(\tau^{\mathrm{p}}-1\right)=\mathrm{M}_{\mathrm{p}} \tau^{\mathrm{p}}=\tau \eta / \epsilon,
$$

from which we conclude that $\epsilon=1$, proving the theorem.

It is clear that Theorem 2 is now essentially proved. The sufficiency was shown in Section 4 and the necessity is a consequence of Theorems 4 and 5 .

\section{CONCLUDING REMARKS.}

We have indicated, in Section 3, that the concept "even integer" was not defined in $\mathrm{F}$ in part because the laws of parity fail to hold in $\mathrm{Q}(\sqrt{-3})$. That is, if one defines $\eta$ to be an even integer iff $\tau \mid \eta$, where $\tau=1+\mathrm{u}$ ( $\mathrm{u}$ a unit in $\mathrm{P}$ ), then the sum of two odd integers may be odd in $\mathrm{Q}(\sqrt{-3})$. (Another reason is that for $\tau=(3+\sqrt{-3}) / 2$, in $\mathrm{Q}(\sqrt{-3}), 2$ is an odd integer!)

Other additive problems in algebraic number theory have involved defining an integer $\eta$ of the field as even if every prime ideal of the first degree which divides the rational prime 2 also divides $\eta$. (In a UFD, this corresponds to (ii) in our listing of alternate definitions of even, in Section 3.) Most notable of these problems are, perhaps, the analogue of the Goldbach conjecture and certain problems involving representations of integers as sums of powers of primes; we refer the interested reader to [13], p. $446^{+}$for a summary of the results on these and related problems.

The analogue of Theorem 1 is provable, except in $\mathrm{F}=\mathrm{Q}(\sqrt{-2})$, under this definition of even integer. (That is, if we define $\tau=1+\mathrm{i}$ for $\mathrm{F}=\mathrm{Q}(\sqrt{-1}), \tau=\sqrt{-2}$ for $\mathrm{F}=\mathrm{Q}(\sqrt{-2})$, and $\tau=2$ for all other fields $\mathrm{F}$, and define $\eta$ to be even iff $\tau \mid \eta$.) However, under the definitions of this paper, $\mathrm{Q}(\sqrt{-2})$ was shown to have no E-perfect numbers (since there exists no prime 
$\tau=1+$ a unit in $Q(\sqrt{-2})$ ), and under these alternate definitions, Theorem 1 is simply not truc for $F=Q(\sqrt{-2})$. This exception points up, again, the observation that the proof in $Q$ of Theorem E-E relies, in an essential way, upon the fact that an even integer has the property that it is divisible not by the "least" prime, but rather, by a prime of the form $1+u$, where $u$ is a unit.

Because many of the results concerning the form or existence of odd perfect numbers in $Q$ are based on the inequality which we have extended to $\mathrm{F}$ in Theorem 3 , it is anticipated that the analogues of these theorems can be obtained in $Q(\sqrt{-1})$ and $Q(\sqrt{-3})$ using the concepts of this paper.

\section{REFERENCES}

1. GEGENBAUER, L., Verslag. Wiss. Ak. Wetenschappe, Amsterdam, 10 (1901-2), 195-207.

2. DICKSON, L. E., History of the Theory of Numbers, Chelsea, New York, 1952.

3. SPIRA, R., "The complex sum of divisors", Amer. Math. Monthly, 68 (1961), 120-124.

4. McDANIEL, W. L., "Perfect Gaussian integers", Acta Arith., 25 (1974), 137-144.

5. GEYER, W.-D., Review of "Perfect ideals over the Gaussian integers", MR 54: 12704.

6. HAUSMAN, M., and SHAPIRO, H. N., "Perfect ideals over the Gaussian integers", Comm. Pure Appl. Math., 29 (1976), 323-341.

7. EULER, L., Opera Postuma 1, Eggers et Socios, Petropoli (Leningrad), 1862.

8. HARDY, G. H. and WRIGHT, E. M., An Introduction to the Theory of Numbers, 4th ed., Clarendon Press, Oxford, 1960.

9. DlCKSON, L. E., Introduction to the Theory of Numbers, University of Chicago Press, 1929.

10. MITRINOVIC, D. S., Elementary Inequalities, Groningen, 1964.

11. RANDALL, L. J., "Perfect Gaussian integers", unpublished manuscript (winning paper in 1980 Science Talent Search). Reviewed in Science News, 117 (1980), 134.

12. GEGENBAUER, L., "Zur Theorie der aus den vierten Einheitswurzeln gebildeten complexen Zahlen", Denkschr. Wien. kㅏ. Wis.

13. NARKIEWICZ, W., Elementary and Analytical Theory of Algebraic Numbers, Polish Scientific Publishers, Warszawa, 1974. 


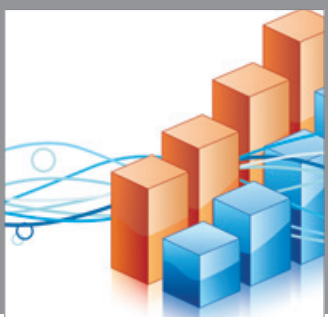

Advances in

Operations Research

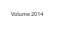

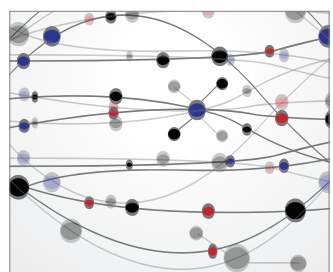

\section{The Scientific} World Journal
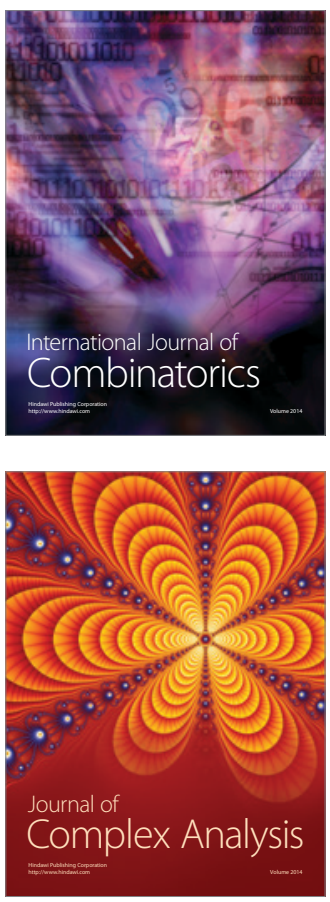

International Journal of

Mathematics and

Mathematical

Sciences
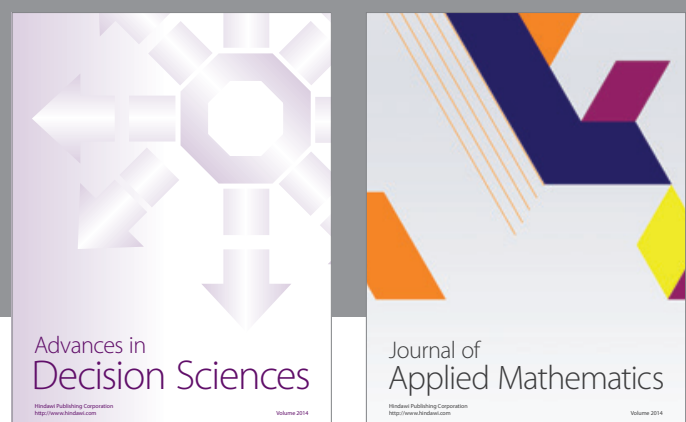

Journal of

Applied Mathematics
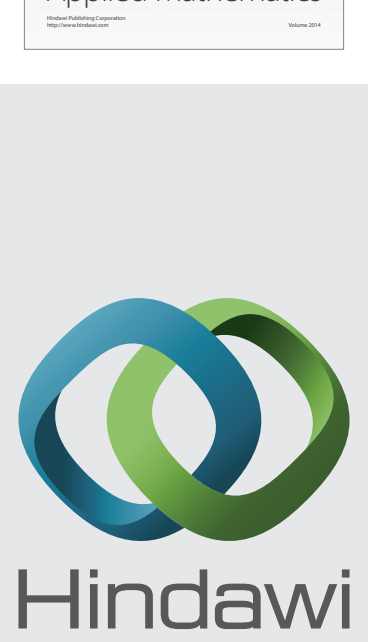

Submit your manuscripts at http://www.hindawi.com
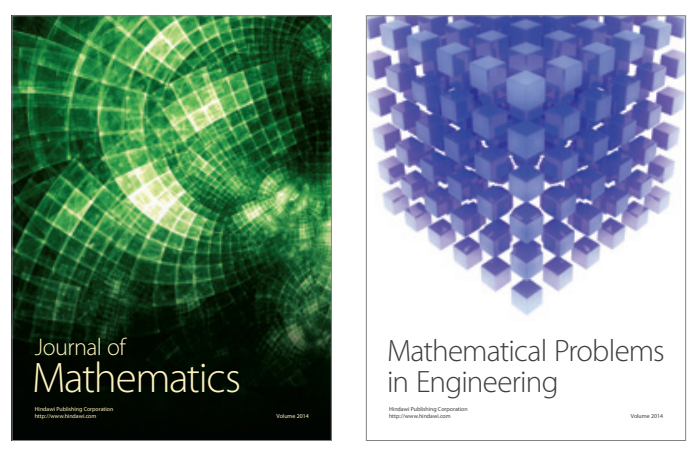

Mathematical Problems in Engineering
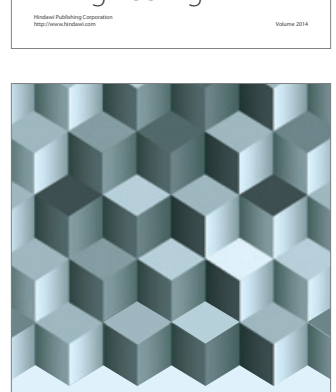

Journal of

Function Spaces
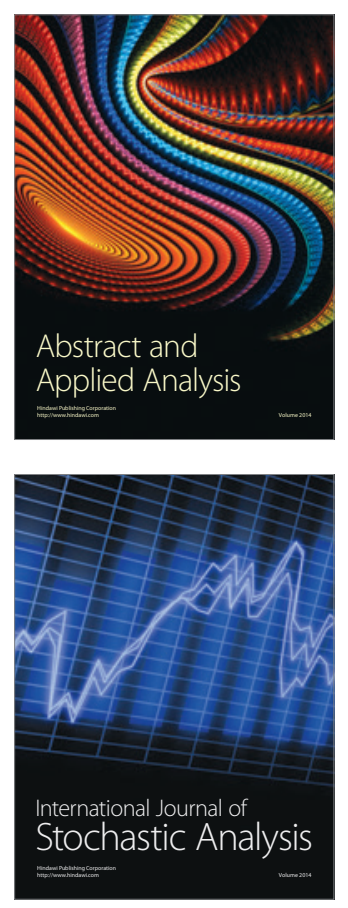

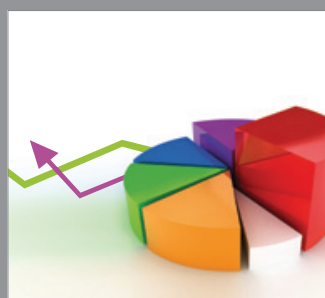

ournal of

Probability and Statistics

Promensencen
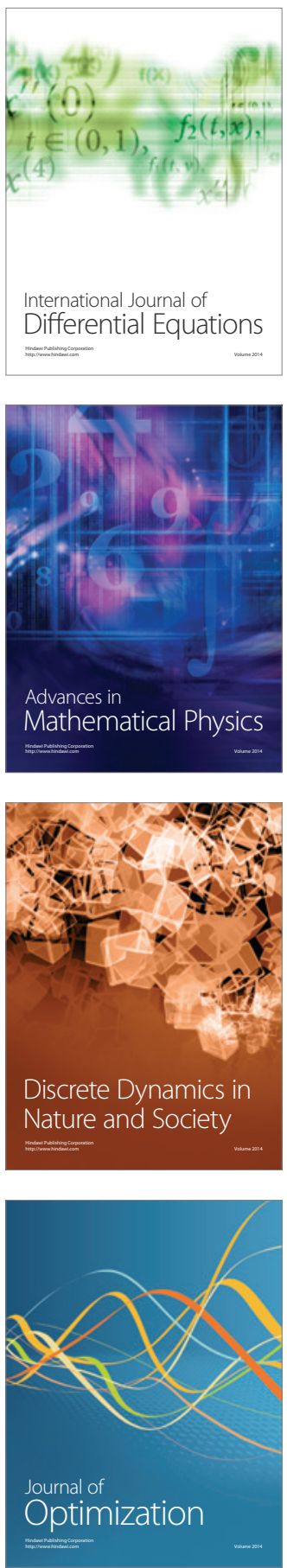